\title{
Learning-based Predictive Beamforming for UAV Communications with Jittering
}

\author{
Weijie Yuan, Member, IEEE, Chang Liu, Member, IEEE, Fan Liu, Member, IEEE, Shuangyang Li, Student \\ Member, IEEE, and Derrick Wing Kwan Ng Senior Member, IEEE
}

\begin{abstract}
As a promising technique for realizing future wireless networks, unmanned aerial vehicle (UAV) communications have drawn numerous attentions. The performance of practical UAV communication systems is limited by the presence of inevitable jittering due to the inherent random wind gusts. The jittering introduces angle ambiguity which is challenging for aligning the information beams between the UAV-mounted base station (BS) and the user equipment (UE). This letter develops a learning-based predictive beamforming scheme to address the beam misalignment caused by UAV jittering. In particular, a deep learning approach is adopted to predict the angles between the UAV and the UE. By doing so, the UAV and the UE can prepare the transmit and receive beams in advance, which enables reliable UAV-based communication. Simulation results verify that the communication performance of the proposed scheme is robust to the presence of UAV jittering.
\end{abstract}

Index Terms-UAV jittering, deep learning, predictive beamforming

\section{INTRODUCTION}

Due to its high flexility and low cost deployment, unmanned aerial vehicle (UAV) systems have a great potential in providing ubiquitous connections for future wireless communication systems [1], [2]. By exploiting the high mobility feature, UAVs can dynamically adjust their locations and area of coverages adapting to the requirement of various practical scenarios, which offers a significant performance improvement compared with terrestrial networks [3], [4]. To support the demand of high data-rate transmission for future wireless communication networks, millimeter wave (mmWave) and massive multi-input multi-output (MIMO) techniques are generally adopted for UAV-based communications, which provide abundant spectral resource, prosperous spatial gain, and highly directional beamforming gain to maintain reliable communication links [5]-[7].

Although the sharp pencil-like spatial beams generated by multi-antenna systems can combat the high path-loss of mmWave signals, it strengthens the requirement of accurately aligning the transmit and receive beams between the UAV and the associated user equipments (UEs). In the literature, the beam alignment problem has been intensively investigated for terrestrial mmWave massive MIMO systems. For instance, in [8], the authors proposed an extended Kalman filtering (EKF)based beam tracking scheme for mmWave systems. However,

This work was supported in part by National Natural Science Foundation of China under Grant 61801082, and in part by Marie Skłodowska-Curie Individual Fellowship under Grant No. 793345. D. W. K. Ng is supported by funding from the UNSW Digital Grid Futures Institute, UNSW, Sydney, under a cross-disciplinary fund scheme and by the Australian Research Council's Discovery Project (DP190101363). (Corresponding author: Chang Liu)

W. Yuan, C. Liu, S. Li, and D. W. K. Ng are with the School of EE\&T, University of New South Wales, NSW 2052, Australia. (e-mail: \{weijie.yuan, chang.liu19, shuangyang.li, w.k.ng\} @unsw.edu.au)

F. Liu is with the Department of Electronic and Electrical Engineering, University College London, London, WC1E 7JE, UK. (e-mail: fan.liu@ucl.ac.uk) this approach is tailored for vehicular networks with only a slight angle variation, which their results are not be applicable to UAV communications with a wide range of angle fluctuations. In [9], the problem of UAV-to-satellite beam alignment was considered, which adopted several sensors mounted on a UAV to acquire the navigation information to assist accurate beam alignment. Nevertheless, such sensors are expensive and unlikely to be deployed on small UAVs, such as civilian drones due to the limited on-board energy supply. Different from terrestrial communication systems, a UAV operating in the air is sensitive to the airflow, which results in random vibrations of the UAV body. This so-called UAV jittering has a significant impact on the communication reliability due to the potential of information beam misalignment [10]. Therefore, it is essential to investigate efficient beam alignment methods for UAV communications with the consideration of jittering.

This paper develops a novel predictive beamforming scheme to address the UAV jittering problem. In particular, we propose to predict the angle at each time slot based on the previous beamforming status. Different from conventional beam alignment that adopts a scanning mechanism, the proposed predictive beamforming scheme allows both the UAV and the UE to adapt their steering beamforming vectors ahead based on the predicted angles for fast link establishment and maintainence. To accurately predict the angles, we propose a deep learning-based scheme, which adopts multiple long shortterm memory (LSTM) layers [11] to facilitate the development of a recurrent neural network (RNN) which can fully exploit the temporal features from the sequential angle data [12]. After receiving the transmitted signals, the UE can further refine the angle and feed it back to the RNN to predict the angle for the next time slot. Benefiting from the powerful deep learning approach, the proposed scheme can accurately predict the angles, which leads to a high beamforming gain and communication rate. Simulation results show that the proposed algorithm can accurately track the beams and maintain reliable communication links even in the presence of UAV jittering.

Notations: The superscripts $(\cdot)^{\mathrm{T}}$, and $(\cdot)^{\mathrm{H}}$ denote the transpose, and the Hermittian operations, respectively; $\mathbb{C}$ denotes the complex space; $\mathcal{C N}(m, v)$ denotes a circular symmetric Gaussian distribution with mean $m$ and variance $v$; $\propto$ represents both sides of the equation are multiplicatively connected to a constant; $\mathcal{O}(\cdot)$ is an asymptotic notation describing the order of complexity; $\mathbb{E}[\cdot]$ denotes the expectation operator; $|\cdot|$ denotes the modulus of a complex number.

\section{SySTEM MODEL}

We consider a downlink UAV-UEcommunication scenario. The UAV transmitter is equipped with $M$ antennas with equal spacing $d_{c}$, forming a uniform linear array (ULA). The UE 


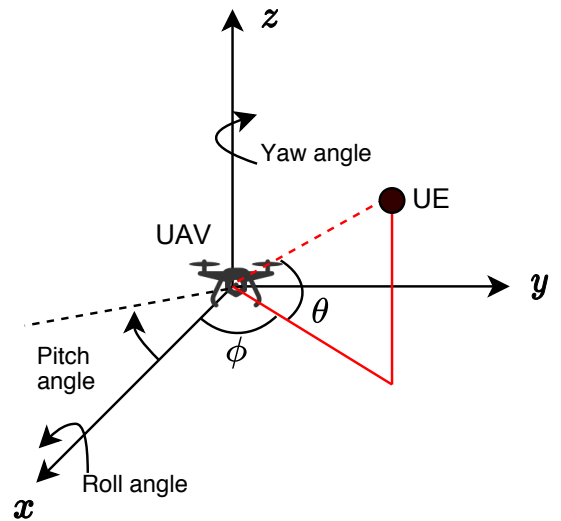

Fig. 1. An illustration of UAV communication scenario.

is equipped with a ULA consisting of $N$ antennas. Following the geometric relationship between the UAV and the UE, we can obtain the azimuth angle, denoted by $\phi$ and the altitude angle, denoted by $\theta$. Since we focus on the impact of UAV jittering, we assume that both locations of the UAV and the UE are fixed.

\section{A. UAV Jittering Model}

Since the UAV hovers in the sky without tieing to some stable infrastructures, random wind gusts can cause UAV jittering [10]. In particular, the UAV may fluctuate in three dimensions, namely, the yaw, the roll, and the pitch motions, as shown in Fig. 1. In general, the UAV vibration is dominated by the pitch motion caused by horizontal airflows [13]. Therefore, in this letter, we only consider the impact of the pitch angle, which is captured by the altitude angle $\theta$. Consequently, we can define the altitude angle at time instant $k$ as

$$
\theta_{k}=\theta_{k-1}+\Delta \theta_{k},
$$

where $\Delta \theta_{k}$ denotes the variation of the altitude angle at time $k$. In general, $\Delta \theta_{k}$ is modeled as a zero mean Gaussian distributed variable with variance $\sigma_{\theta}^{2}$. The value of $\sigma_{\theta}$ depends on the wind speed, typically in the range of $[0.01,0.1] \mathrm{rad}$ [13]. In general, the jittering follows some patterns despite the randomness in the airflow. For instance, the wind direction is unlikely to change to the opposite direction in a short time. Also, the wind speed does not have significant variations in a short time period. Exploiting these characteristics, we further assume that $\sigma_{\theta}$ is time varying and the coherence time of $\sigma_{\theta}$ is $T_{\sigma}$, i.e., $\sigma_{\theta}$ changes its value per $T_{\sigma}$ sampling periods [12].

\section{B. Communication Model}

We assume that the UAV communicates with the UE via a line-of-sight (LoS) channel ${ }^{1}$. Based on the above discussions, we see that both angle-of-arrival (AoA) and angle-of-departure (AoD) experience the same fluctuations due to the UAV jittering, therefore we assume that the AoAs and AoDs are

\footnotetext{
${ }^{1}$ Since the rich scattering scenario rarely appears in mmWave UAV communications, we assume that the LoS propagation dominates the communication link, as commonly adopted in the literature [14], [15].
}

identical to the altitude angles. In particular, the channel matrix $\mathbf{H} \in \mathbb{C}^{N \times M}$ can be expressed as [14]

$$
\mathbf{H}=\frac{\lambda_{c}}{4 \pi d_{0}} \mathbf{b}\left(\theta_{k}\right) \mathbf{a}\left(\theta_{k}\right)^{\mathrm{H}},
$$

where $\lambda_{c}$ is the wavelength of the carrier frequency and $d_{0}$ is the range distance between the UAV and the UE. As we assume fixed positions for the UAV and the UE, the distance $d_{0}$ is a constant. For notational simplicity, we use $h_{0}=\frac{\lambda_{c}}{4 \pi d_{0}}$ to denote the average path gain between the UAV and the UE. Without loss of generality, the transmit and receive steering vectors are given by

$$
\begin{aligned}
\mathbf{a}\left(\theta_{k}\right) & =\sqrt{\frac{1}{M}}\left[1, e^{-j \frac{2 \pi d_{c} \cos \theta_{k}}{\lambda_{c}}}, \ldots, e^{-j \frac{2 \pi d_{c}(M-1) \cos \theta_{k}}{\lambda_{c}}}\right]^{\mathrm{T}}, \\
\mathbf{b}\left(\theta_{k}\right) & =\sqrt{\frac{1}{N}}\left[1, e^{-j \frac{2 \pi d_{c} \cos \theta_{k}}{\lambda_{c}}}, \ldots, e^{-j \frac{2 \pi d_{c}(N-1) \cos \theta_{k}}{\lambda_{c}}}\right]^{\mathrm{T}},
\end{aligned}
$$

respectively, where $d_{c}$ is the antenna spacing. Note that we can generally choose $\lambda_{c}=2 d_{c}$ to further simplify the expressions of $\mathbf{a}\left(\theta_{k}\right)$ and $\mathbf{b}\left(\theta_{k}\right)$.

At time instant $k$, the UAV transmits a sequence of symbols $\mathbf{s}_{k}=\left[s_{k, 1}, \ldots, s_{k, L}\right]$ to the UE. The first symbol of $\mathbf{s}_{k}$, i.e., $s_{k, 1}$ is a pilot to assist the beam alignment. For each transmitted symbol $s_{k, l}$, it is multiplexed over $M$ transmit antennas using beamforming vector $\mathbf{w}_{k} \in \mathbb{C}^{M \times 1}$. After transmitting through the channel, the post processed signal $r_{k} \in \mathbb{C}$ at the UE is given by

$$
r_{k, l}=\mathbf{f}_{k}^{\mathrm{H}} \mathbf{H} \mathbf{w}_{k} s_{k, l}+z_{k},
$$

where $\mathbf{f}_{k} \in \mathbb{C}^{N \times 1}$ is the receive beamforming vector and $z_{k} \in$ $\mathbb{C}$ is the complex noise term obeying $\mathcal{C N}\left(0, \sigma^{2}\right)$ at the UE. Based on (3), the receive signal-to-noise ratio (SNR) at the UE at time instant $k$ is given by $\mathbb{E}\left[\left|\mathbf{f}_{k}^{\mathrm{H}} \mathbf{H} \mathbf{w}_{k} s_{k, l}\right|^{2}\right] / \sigma^{2}$. Assuming that the transmit signal has a unit power, i.e., $\mathbb{E}\left[\left|s_{k, l}\right|^{2}\right]=1$, and substituting the expression of $\mathbf{H}=h_{0} \mathbf{b}\left(\theta_{k}\right) \mathbf{a}\left(\theta_{k}\right)^{\mathrm{H}}$, the receive SNR is expressed as

$$
\mathrm{SNR}_{k}=\frac{\left|h_{0} \mathbf{f}_{k}^{\mathrm{H}} \mathbf{b}\left(\theta_{k}\right) \mathbf{a}\left(\theta_{k}\right)^{\mathrm{H}} \mathbf{w}_{k}\right|^{2}}{\sigma^{2}}
$$

and the corresponding achievable rate $R_{k}$ is

$$
R_{k}=\log _{2}\left(1+\mathrm{SNR}_{k}\right) .
$$

It can be observed that the designed beamformers $\mathbf{w}_{k}$ and $\mathbf{f}_{k}$ should be dependent on the estimated altitude angle $\theta_{k}$. Due to the narrow beamwidth in massive antenna systems, even a slight variation of $\Delta \theta_{k}$ would result in severe beam misalignment and lead to dramatic communication performance degradation. As a remedy, we will develop a fast beam alignment scheme in what follows.

\section{Fast Beam Alignment Algorithm}

To address the beam alignment caused by jittering of the UAV, we develop a predictive beamforming algorithm. In particular, the proposed algorithm consists of a prediction step and a refinement step. Given the refined angles of the previous time instants, the angle at the current instant can be predicted using the proposed deep learning approach. Specifically, the 


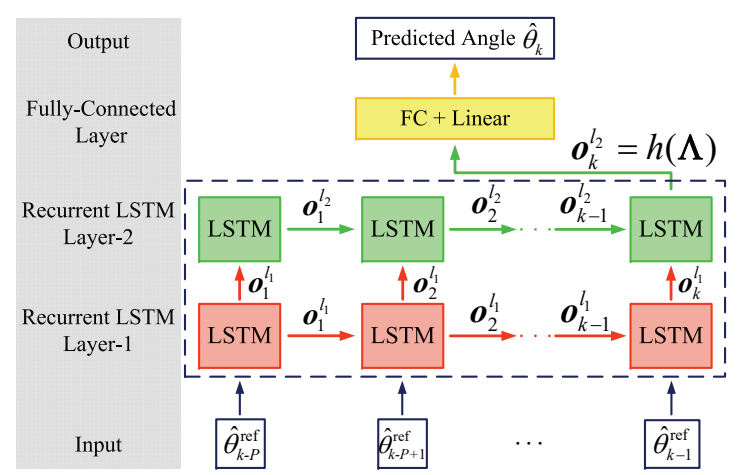

Fig. 2. The developed LSTM-RNN architecture for angle prediction. The horizontal arrows represent the communications from one time-step to the other, meanwhile the up pointing arrows represent the communications from the current layer to the next layer.

predicted angle is exploited for designing the beamforming vectors. Then after signal transmission, the predicted angle can be further refined by the received downlink pilot symbol. Finally, the refined angle is fed to the RNN to predict the angle at the next instant.

\section{A. Deep RNN-based Beam Prediction}

Here, we design an LSTM-based RNN (LSTM-RNN) for the angle prediction. The detailed architecture of the proposed LSTM-RNN is illustrated in Fig. 2, which consists of two LSTM layers and one fully-connected (FC) layer. The LSTM layers are adopted to learn the long-term dependence property of the input angle sequences and the FC layer is introduced to linearly combine the learned features to further improve the performance. The hyperparameters are introduced in Table I, where "Linear" represents the linear activation function [16].

TABLE I

HYPERPARAMETERS OF THE PROPOSED LSTM-RNN

\begin{tabular}{cc}
\hline Hyperparameters & Values \\
\hline Output dimension of LSTM layer-1 $\left(\mathbf{o}_{t}^{l_{1}}\right)$ & 50 \\
\hline Output dimension of LSTM layer-2 $\left(\mathbf{o}_{t}^{l_{2}}\right)$ & 100 \\
\hline Number of time steps for each LSTM layer & $k$ \\
\hline Output dimension of FC layer & 1 \\
\hline Activation function of FC layer & Linear \\
\hline
\end{tabular}

LSTM layer-1 is a recurrent layer which adopts the same LSTM structure for each time step. Denote by $\mathbf{o}_{t}^{l_{i}}$ the output of LSTM layer- $i, i=\{1,2\}$, at the $t$-th, $t=\{1,2, \cdots, K\}$, time step. For LSTM layer-1, LSTM at each time step passes the output $\mathbf{o}_{t}^{l_{1}}$ to the next layer and keeps a copy to itself for the calculation at next time step. For LSTM layer-2, LSTM only keeps the output $\mathbf{o}_{t}^{l_{2}}$ to itself for the previous $P$ time steps and finally passes $\mathbf{o}_{k}^{l_{2}}$ to the FC layer at the $k$-th time step. Given an angle sequence $\boldsymbol{\Lambda}=\left\{\hat{\theta}_{K-P}^{\mathrm{ref}}, \hat{\theta}_{k-P+1}^{\mathrm{ref}}, \cdots, \hat{\theta}_{k-1}^{\mathrm{ref}}\right\}$, if we regard both LSTM layer-1 and LSTM layer-2 as an LSTM block denoted by a function $h(\cdot)$, the output of the LSTM block is given by

$$
\mathbf{o}_{k}^{l_{2}}=h(\boldsymbol{\Lambda}) .
$$

As shown in Fig. 2, the output $\mathbf{o}_{k}^{l_{2}}$ is then sent to the next FC layer and we can finally obtain the output. Let $\mathcal{R}_{w}(\cdot)$ denote the LSTM-RNN where $w$ is the total network parameter, the output of LSTM-RNN can be expressed as

$$
\hat{\theta}_{k}=\mathcal{R}_{w}(\boldsymbol{\Lambda})=\sigma(\mathbf{W} h(\boldsymbol{\Lambda})+\mathbf{b}),
$$

where $\hat{\theta}_{k}$ is the predicted angle value for time instant $k, \sigma(\cdot)$ denotes the linear activation function, and $\mathbf{W} \in \mathbb{C}^{1 \times 100}$ and $\mathbf{b} \in \mathbb{C}^{1 \times 100}$ are the weights and bias of FC layer, respectively.

Since the prediction problem is a regression task, we select a mean squared error (MSE) cost function for our proposed LSTM-RNN, which is defined by [16]

$$
J_{\mathrm{MSE}}(w)=\frac{1}{2 Q} \sum_{q=1}^{Q}\left(\theta_{k}^{(q)}-\sigma\left(\mathbf{W} h\left(\boldsymbol{\Lambda}^{(q)}\right)+\mathbf{b}\right)\right)^{2},
$$

where $\theta_{k}^{(q)}$ and $\boldsymbol{\Lambda}^{(q)}$ are the label and input of the $q$-th, $q \in$ $\{1,2, \cdots, Q\}$, training example, respectively. Based on (8), we can then use a backpropagation algorithm to update the network parameters progressively and finally obtain the welltrained LSTM-RNN for angle prediction.

Generally, the MSE [16] is a common adopted metric to characterize the relationship between the predictive quantity and the actual quantity in the LSTM-RNN model. Specifically, the MSE between the predicted altitude angle $\hat{\theta}_{k}$ and the actual altitude angle $\theta_{k}$ is defined as

$$
\mathrm{MSE}=\mathbb{E}\left[\left|\hat{\theta}_{k}-\theta_{k}\right|^{2}\right] .
$$

The smaller the value of MSE is, the closer the predicted altitude angle approaches to the actual altitude angle.

After training process, we can obtain the well-trained LSTM-RNN and the predictive angle $\hat{\theta}_{k}$ can be expressed as

$$
\hat{\theta}_{k}=\mathcal{R}_{w^{*}}(\boldsymbol{\Lambda}) \text { with } w^{*}=\arg \min _{w} J_{\mathrm{MSE}}(w),
$$

where $w^{*}$ is the well-trained network parameter and $\boldsymbol{\Lambda}$ denotes the input sequence. Note that when the number of training examples are large enough in capturing the various impacts of natural factors on jittering, the cost function in (8) approaches the MSE metric in (9). Therefore, the predictive quantity is able to approach the actual quantity arbitrary close as long as the training set is sufficiently large.

Having obtained the predicted angle $\hat{\theta}_{k}$ at the UE, it feeds back the predicted angle to the UAV via an out-of-band communication uplink. Relying on powerful channel coding techniques, the UAV can accurately acquire the predicted angle, as commonly adopted in [8]. Then the UAV and the UE can easily formulate their beamforming vectors for downlink communication at time $k$, i.e, $\mathbf{w}_{k}=\mathbf{a}\left(\hat{\theta}_{k}\right)$ and $\mathbf{f}_{k}=\mathbf{b}\left(\hat{\theta}_{k}\right)$ and establish the communication link ${ }^{2}$. Besides, during the data transmission stage, the UAV sends a pilot symbol $s_{k, 1}$ to the UE for refining the predicted angle. Note that when the predicted angle $\hat{\theta}_{k}$ equals to the actual angle $\theta_{k}$, the achievable rate $R_{k}$ is maximized.

\footnotetext{
${ }^{2}$ To avoid any possible impact due to communication delay in feedback links, the UE can perform a two-step prediction to obtain the angle $\hat{\theta}_{k+1}$ using the RNN and feeds it back to the UAV at time $k-1$. Hence, at time $k+1$, the UAV can formulate its transmit beamformers using $\hat{\theta}_{k+1}$ despite the existence of possible communication delay.
} 


\section{B. Refinement of $\hat{\theta}_{k}$ in Real Time}

The predicted angle $\hat{\theta}_{k}$ is refined at the UE based on the received pilot symbol $s_{k, 1}$ in (3) by adopting the maximum likelihood estimator, which is given by

$$
\hat{\theta}_{k}^{\mathrm{ref}}=\arg \max _{\theta_{k}} p\left(r_{k, 1} \mid \theta_{k}\right)=\arg \max _{\theta_{k}} \ln p\left(r_{k, 1} \mid \theta_{k}\right),
$$

where $p\left(r_{k} \mid \theta_{k}\right) \propto \exp \left(-\left|r_{k}-\mathbf{f}_{k}^{\mathrm{H}} \mathbf{H} \mathbf{w}_{k} s_{k, 1}\right|^{2} / 2 \sigma^{2}\right)$. Obviously, the maximization is achieved when $\theta_{k}$ satisfying $r_{k, 1}=$ $\mathbf{f}_{k}^{\mathrm{H}} \mathbf{H} \mathbf{w}_{k} s_{k, 1}$. With $\mathbf{w}_{k}=\mathbf{a}\left(\hat{\theta}_{k}\right)$ and $\mathbf{f}_{k}=\mathbf{b}\left(\hat{\theta}_{k}\right)$, our goal is to infer $\theta_{k}$ from the equation

$$
r_{k}=\frac{1}{M N} h_{0} s_{k, 1} \sum_{n=1}^{N} \sum_{m=1}^{M} e^{j(m-n) \pi\left(\cos \theta_{k}-\cos \hat{\theta}_{k}\right)} .
$$

The nonlinearity in (12) makes the closed-form solution for $\theta_{k}$ infeasible. Therefore, we resort to the first-order Taylor expansion to overcome the nonlinearity. By letting $f\left(\theta_{k}\right)=\sum_{n=1}^{N} \sum_{m=1}^{M} e^{j(m-n) \pi\left(\cos \theta_{k}-\cos \hat{\theta}_{k}\right)}$, the first-order Taylor series around the predicted angle $\hat{\theta}_{k}$ is given by $f\left(\theta_{k}\right) \approx f\left(\hat{\theta}_{k}\right)+f^{\prime}\left(\hat{\theta}_{k}\right)\left(\theta_{k}-\hat{\theta}_{k}\right)$, where $f^{\prime}\left(\hat{\theta}_{k}\right)$ denotes the derivative of $f\left(\theta_{k}\right)$ evaluated at the point of $\hat{\theta}_{k}$. After some manipulations, we have the approximation of $f\left(\theta_{k}\right) \mathrm{as}^{3}$

$$
f\left(\theta_{k}\right) \approx M N-j \pi \frac{M N(M-N)}{2} \sin \hat{\theta}_{k}\left(\theta_{k}-\hat{\theta}_{k}\right) .
$$

With the approximated version of $f\left(\theta_{k}\right)$, we can easily obtain the refined angle $\hat{\theta}_{k}^{\text {ref }}$ at time $k$, which is given by

$$
\hat{\theta}_{k}^{\mathrm{ref}}=\hat{\theta}_{k}+\frac{2 h_{0} s_{k, 1}-2 r_{k, 1}}{h_{0} s_{k, 1} j \pi(M-N) \sin \hat{\theta}_{k}},
$$

where the second term on the right hand side denotes the refinement term based on the received signal sample. Specifically when the beams are perfectly aligned, we have $r_{k, 1}=$ $h_{0} s_{k, 1}+z_{k}$ and refinement term only regulates the impact due to noise. After obtaining the refined angle $\hat{\theta}_{k}^{\text {ref }}$, we can feed it to the RNN and predict the altitude angle $\hat{\theta}_{k+1}$ at time $k+1$.

\section{Complexity Analysis of the Proposed LSTM-RNN Method}

According to [11] and [17], the time complexity of an LSTM per time step is

$$
\mathcal{O}\left(4\left(m n+n^{2}+n\right)\right)
$$

where $m$ and $n$ denotes the input dimension and output dimension, respectively. Note that the proposed LSTM-RNN model has two layers and $K$ time steps. Let $m_{i}$ and $n_{i}$, $i \in\{1,2\}$, denote the input dimension and output dimension of the LSTM layer- $i$ in the proposed LSTM-RNN model, respectively. In addition, we use terms $N_{t}$ and $N_{e}$ to represent the numbers of training examples and epoches of the offline training, respectively.

\footnotetext{
${ }^{3}$ The first-order Taylor expansion suffers from some approximation errors due to neglecting the higher order terms. However, the powerful RNN-based prediction can take the error into account and adjust the prediction accordingly. Moreover, when $M=N, f\left(\theta_{k}\right)$ is a constant and the refined angle is identical to the predicted angle and thus no refinement is performed.
}

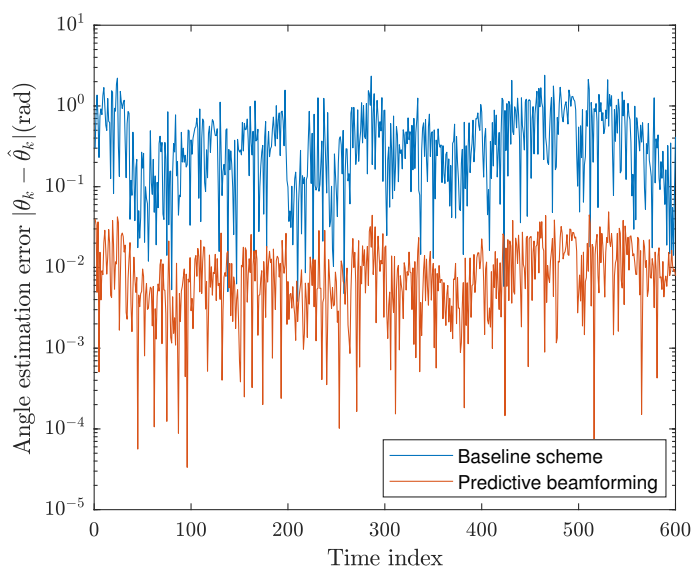

Fig. 3. The angle estimation error versus time.

The time complexity of offline training using the proposed LSTM-RNN method is derived as

$$
C_{\text {training }}=\mathcal{O}\left(4 K N_{t} N_{e}\left(\sum_{i=1}^{2} m_{i} n_{i}+n_{i}^{2}+n_{i}\right)\right) .
$$

Moreover, the complexity of online prediction is given by

$$
C_{\text {prediction }}=\mathcal{O}\left(4 K\left(\sum_{i=1}^{2} m_{i} n_{i}+n_{i}^{2}+n_{i}\right)\right),
$$

which is in polynomial time and suitable for real-time implementation [18].

\section{Simulation Results}

In this section, we verify our proposed algorithm through numerical simulations. We assume that the UAV has $M=64$ antennas and the UE has $N=32$ antennas. The UAV-UE system operates at a carrier frequency of $60 \mathrm{GHz}$ and range distance between the UAV and the UE is $150 \mathrm{~m}$. The UAV transmit power is set as $30 \mathrm{dBm}$. Without loss of generality, the initial altitude angle is $1 \mathrm{rad}$. The time duration for a single slot is $\Delta T=0.02$ second $^{4}$. For modeling the UAV jittering, we set the coherence time of wind gusts as $T_{\sigma}=20 \Delta T$ and the standard derivation of the angle variation as $\sigma_{\theta} \in[0.01,0.02]$ rad. In addition, the hyperparameters of our proposed LSTMRNN are listed in Table I with $k=19$. There are 20,000 examples in the training set which are stored at the UE. The neural network model is trained offline and the prediction process is done at the UE. Finally, the thermal noise power $\sigma^{2}$ is $-90 \mathrm{dBm}$.

In Fig. 3, we evaluate the tracking performance of the proposed deep learning-based predictive beamforming scheme and a baseline scheme. The angle error is defined by the absolute value of $\theta_{k}-\hat{\theta}_{k}$. The baseline scheme directly utilizes the actual angle from the previous time slot to formulate the beamformers in the current time instant. It can be observed that the predicted angle can accurately track the variation of the actual angle, while the angle error becomes exceedingly

\footnotetext{
${ }^{4}$ As we consider fixed positions for UAV and UE, the coherence time for the UAV-to-UE channel is much larger than $0.02 \mathrm{~s}$. Therefore, we assume that the channel gain $h_{0}$ remains constant in the considered simulations.
} 


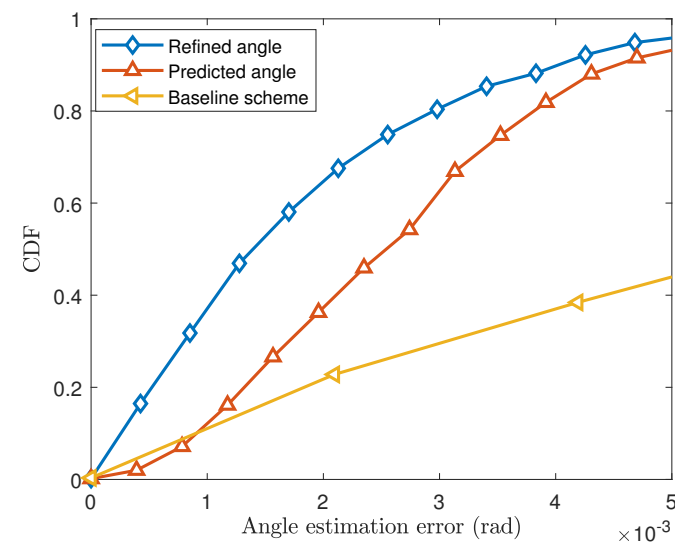

Fig. 4. The CDF of angle estimation error.

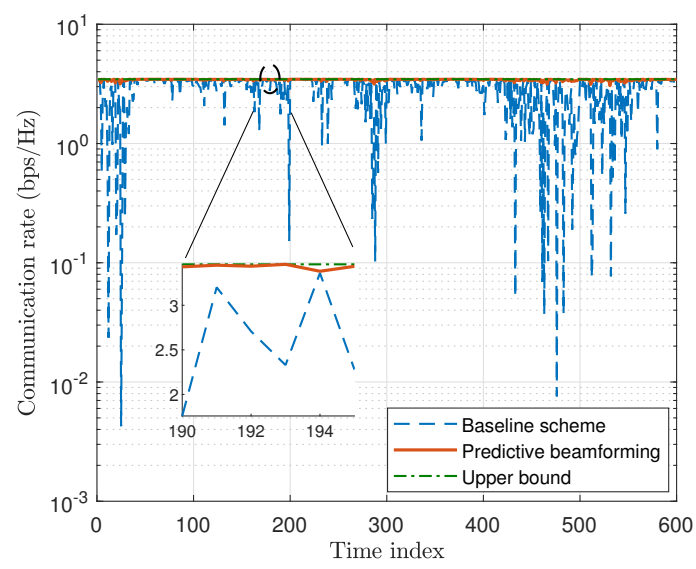

Fig. 5. The comparison of the communication rate between the proposed algorithm and different schemes.

large for the baseline scheme at some time slots when the angle varies drastically. The simulation results also show that the angle error between the predicted altitude angle of the proposed LSTM-RNN method and the actual altitude angle is around $10^{-3} \mathrm{rad}$ which verifies the high accuracy of the proposed method. Next, we compare the cumulative distribution function (CDF) of the angle estimation error in all time slots between the proposed algorithm and the baseline scheme in Fig. 4. It can be seen that both the predicted angle and the refined angle obtained with the first-order Taylor expansion achieve an estimation error at $10^{-3}$ rad level, while the baseline suffers from significant performance loss. This validates the benefits of applying the proposed predictive beamforming design.

In Fig. 5, we compare the communication rates of the proposed algorithm and the baseline scheme. The communication rate obtained by assuming the availability of actual angles is depicted as a performance upper bound. It can be seen that the communication link between the UAV and the UE established by the proposed predictive beamforming scheme is robust to UAV jittering. In particular, only a slight degradation of the communication rate can be observed compared with the upper bound. In contrast, for the baseline scheme, when the angle varies violently, the communication rate degrades and fluctuates significantly. This provides a clear evidence on the superiority of the proposed technique over the baseline scheme.

\section{CONCLUSIONS}

In this letter, we proposed a novel deep learning-based predictive beamforming scheme in the presence of UAV jittering. We trained the prediction model by adopting a RNN to exploit the temporal features from the sequential angle data. Then the altitude angle at the current time slot was predicted using the angle estimates in the previous slots. The beamformers were then designed to estabilish the communication link. Simulation results showed that our proposed scheme can accurately track the variation of the angles. Moreover, the communication performance significantly outperforms the baseline scheme in the presence of UAV jittering.

\section{REFERENCES}

[1] V. W. Wong, R. Schober, D. W. K. Ng, and L.-C. Wang, Key Technologies for $5 G$ Wireless Systems. Cambridge university press, 2017.

[2] X. Liu and N. Ansari, "Resource allocation in UAV-assisted M2M communications for disaster rescue," IEEE Wireless Commun. Lett., vol. 8, no. 2, pp. 580-583, Apr. 2018.

[3] Y. Zeng, Q. Wu, and R. Zhang, "Accessing from the sky: A tutorial on UAV communications for 5G and beyond," Proc. IEEE, vol. 107, no. 12, pp. 2327-2375, Dec. 2019.

[4] T. Zhang, Y. Xu, J. Loo, D. Yang, and L. Xiao, "Joint computation and communication design for UAV-assisted mobile edge computing in IoT," IEEE Trans. Ind. Inform., vol. 16, no. 8, pp. 5505-5516, Aug. 2019.

[5] Q. Wu, L. Liu, and R. Zhang, "Fundamental trade-offs in communication and trajectory design for UAV-enabled wireless network," IEEE Wireless Commun., vol. 26, no. 1, pp. 36-44, Jan. 2019.

[6] N. Zhao, Y. Li, S. Zhang, Y. Chen, W. Lu, J. Wang, and X. Wang, "Security enhancement for NOMA-UAV networks," IEEE Trans. Veh. Technol., vol. 69, no. 4, pp. 3994-4005, Apr. 2020.

[7] N. Zhao, F. Cheng, F. R. Yu, J. Tang, Y. Chen, G. Gui, and H. Sari, "Caching UAV assisted secure transmission in hyper-dense networks based on interference alignment," IEEE Trans. Commun., vol. 66, no. 5, pp. 2281-2294, May 2018.

[8] S. Shaham, M. Ding, M. Kokshoorn, Z. Lin, S. Dang, and R. Abbas, "Fast channel estimation and beam tracking for millimeter wave vehicular communications," IEEE Access, vol. 7, pp. 141 104-141 118, 2019.

[9] L. Yang and W. Zhang, "Beam tracking and optimization for UAV communications," IEEE Trans. Wireless Commun., vol. 18, no. 11, pp. 5367-5379, Nov. 2019.

[10] D. Xu, Y. Sun, D. W. K. Ng, and R. Schober, "Multiuser MISO UAV communications in uncertain environments with no-fly zones: Robust trajectory and resource allocation design," IEEE Trans. Commun. vol. 68, no. 5, pp. 3153-3172, May 2020.

[11] S. Hochreiter and J. Schmidhuber, "Long short-term memory," Neural Comput., vol. 9, no. 8, pp. 1735-1780, Nov. 1997.

[12] R. Pascanu, C. Gulcehre, K. Cho, and Y. Bengio, "How to construct deep recurrent neural networks," in Proc. IEEE Int. Conf. Learn. Representations (ICLR). Banff, AB, Canada, Apr. 2014.

[13] M. Ryll, H. H. Bülthoff, and P. R. Giordano, "A novel overactuated quadrotor unmanned aerial vehicle: Modeling, control, and experimental validation," IEEE Trans. Control Syst. Technol., vol. 23, no. 2, pp. 540556, Feb. 2014.

[14] J. Zhao, F. Gao, L. Kuang, Q. Wu, and W. Jia, "Channel tracking with flight control system for UAV mmWave MIMO communications," IEEE Commun. Lett., vol. 22, no. 6, pp. 1224-1227, Jun. 2018.

[15] Y. Cai, Z. Wei, R. Li, D. W. K. Ng, and J. Yuan, "Joint trajectory and resource allocation design for energy-efficient secure UAV communication systems," IEEE Trans. Commun., 2020, in press.

[16] I. Goodfellow, Y. Bengio, A. Courville, and Y. Bengio, Deep learning. MIT Press Cambridge, 2016.

[17] E. Tsironi, P. Barros, C. Weber, and S. Wermter, "An analysis of convolutional long short-term memory recurrent neural networks for gesture recognition," Neurocomputing, vol. 268, pp. 76-86, Dec. 2017.

[18] T. H. Cormen, C. E. Leiserson, R. L. Rivest, and C. Stein, Introduction to algorithms. MIT press, 2009. 